\title{
A spectrophotometric catalogue of HII galaxies ${ }^{\star}$
}

\author{
C.C. Popescu ${ }^{1,2}$ and U. Hopp ${ }^{3}$ \\ 1 Max Planck Institut für Kernphysik, Saupfercheckweg 1, D-69117 Heidelberg, Germany \\ 2 The Astronomical Institute of the Romanian Academy, Str. Cuţitul de Argint 5, 75212 Bucharest, Romania \\ 3 Universitätssternwarte München, Scheiner Str. 1, D-81679 München, Germany
}

Received October 25; accepted December 1, 1999

\begin{abstract}
We present a spectrophotometric catalogue of 90 emission-line galaxies (ELGs) discovered during an objective - prism survey that aimed to search for dwarf galaxies within the voids (Popescu et al. 1996, 1998). The paper presents line ratios, equivalent widths and absolute fluxes for the emission-lines seen in the spectra of the galaxies. A list of newly discovered Wolf-Rayet galaxies is presented. Many objects included in the catalogue have low metallicity and the extreme cases are proposed as candidates for very low metallicity galaxies.
\end{abstract}

Key words: galaxies: compact - galaxies: dwarf galaxies: abundances — galaxies: statistics — galaxies - distances and redshifts - large scale structure of Universe

\section{Introduction}

Popescu et al. (1996, 1998) conducted a survey for emission-line galaxies (ELGs) based on the Hamburg Quasar Survey (HQS, Hagen et al. 1995) - IIIa-J digitised objective prism plates. The main goal of the project was to search for dwarf ELGs in voids and to analyse the large-scale structure of the distribution of this kind of galaxies (Popescu et al. 1997). The follow-up spectroscopy at the $2.2 \mathrm{~m}$ telescope at the German-Spanish Observatory at Calar Alto (Almeria, Spain) provided us with a complete sample of 250 ELGs, of which many are Blue Compact Dwarfs (BCDs) or HII galaxies. These two terms are usually used to define the same kind of objects. While the name BCD was mainly used for objects classified on morphological criteria (Binggeli et al. 1985 for the Virgo Cluster Catalogue), the term of HII galaxy

Send offprint requests to: C.C. Popescu

e-mail: popescu@levi.mpi-hd.mpg.de

* Based on observations obtained at the German-Spanish Observatory at Calar Alto, Almeria, Spain. was introduced for objects discovered on spectroscopic surveys for emission-line galaxies. These objects have focused attention after the discovery by Sargent \& Searle (1970) that some of them were low metallicity systems hosting a very active stellar formation. Gallagher \& Hunter (1989) showed that the BCDs present also the lowest mass surface density and rotation velocities ever measured for objects supporting star formation activity. Thus it was suggested (Vilchez 1995) that these galaxies can be easily affected by environmental factors. Since in our study of the spatial distribution of ELGs (Popescu et al. 1997) we identified the isolation of each sample galaxy, we can address the question of environmental influences on the star-forming properties of HII galaxies, using an unbiased sample. In this paper we give the catalogue of the spectroscopic parameters of a subsample of 90 ELGs and we describe the data sample. The influence of the environment on the mechanism that controls star formation is discussed in a separate paper (Popescu et al. 1999).

\section{The data}

From our sample of 250 ELGs we selected for analysis only objects from a region North of the "Slice of the Universe" (de Lapparent et al. 1986), which is dominated by very well defined nearby voids. This subsample consists of 90 ELGs, most of them being distributed in the sheets and filaments that surround the voids, and with a few ELGs within some nearby voids or at the rim of the voids. No rich cluster of galaxies was included in the survey.

Most of the galaxies from this sample were observed twice. The objects were first observed in a snap-shot mode (Popescu et al. 1997), in order to check the selection criteria and to measure redshifts. In these campaigns we mostly did not have photometric conditions, therefore no reliable absolute fluxes could have been derived. Also a slit width of $2^{\prime \prime}$ was used, which did not provide a total coverage of the galaxies. The observing campaign from May 1997 (see 
Table 1. The details of the spectroscopic observations from May 1996

\begin{tabular}{ll}
\hline \hline & \\
Detector & Lor-80 \\
Pixel Size $(\mu)$ & 15 \\
Slit width $($ II) & 4 \\
PA & 90. \\
Pixel number & $1024 \times 1024$ \\
Grism & 9 \\
Dispersion $(\AA /$ pixel $)$ & 5.6 \\
Resolution $(\AA)$ & 17 \\
Spectral Range $(\AA)$ & $3600-9000$ \\
& \\
\hline
\end{tabular}

Table 2.

\begin{tabular}{|c|c|}
\hline error & emission line flux or equivalent width \\
\hline $5 \%$ & $\begin{array}{l}\mathrm{H} \beta,[\mathrm{OIII}] \lambda 4959,[\mathrm{OIII}] \lambda 5007, \mathrm{H} \alpha \\
W([\mathrm{OIII}]), W(\mathrm{H} \alpha)\end{array}$ \\
\hline $10 \%$ & {$[\mathrm{OII}] \lambda 3727, \mathrm{H} \gamma, \mathrm{HeI} \lambda 5876, W(\mathrm{H} \beta)$} \\
\hline $15 \%$ & $\mathrm{H} \delta,[\mathrm{SII}] \lambda 6717,6731$ \\
\hline $20 \%$ & $\begin{array}{l}{[\mathrm{OI}] \lambda 6300, \mathrm{HeI} \lambda 6678, \mathrm{HeII} \lambda 4686,} \\
W([\mathrm{SII}])\end{array}$ \\
\hline $25 \%$ & $\operatorname{HeI} \lambda 7065,[\mathrm{AIII}] \lambda 7136$ \\
\hline $30 \%$ & {$[\mathrm{OI}] \lambda 6364$} \\
\hline
\end{tabular}

Table 1 for the details of the observations) was dedicated to the spectrophotometry of our objects, but it also completed the observations of selected candidates. These latter observations were done in good photometric conditions, with a slit width of $4^{\prime \prime}$. For the small projected sizes of our dwarf galaxies, such an aperture is large enough to encompass most of their line emission. For the purpose of estimating the fraction of the line emission included in the slit we list in all the relevant tables of the paper the seeing-corrected angular sizes of the star-forming HII regions $\left(r_{\mathrm{HII}}\right)$. The comparison between the angular sizes of the emitting regions and the aperture sizes lead to the conclusion that the fluxes measured in the campaign from May 1996 represent accurate measurements of the total integrated fluxes of our galaxies. The large aperture used to obtain total fluxes reduces the resolution of our spectra, therefore any deblending of the emission lines was performed on the spectra taken in the previous campaigns. For the few galaxies observed only in the May 1997 observing campaign, no deblending was performed on the lines. Also, for the galaxies observed only in non-photometric conditions, no fluxes were assigned, only relative line ratios.

The data were reduced using the MIDAS routines (as described by Popescu et al. 1996, 1998). The photometric calibrations of the spectra taken in the May 97 observing run were done using the standard stars: HZ 21, HZ 44 and $\mathrm{BD}+33^{\circ} 2642$ (Oke 1990) and the absolute fluxes are accurate to about $10 \%$. For one observing night the accuracy of the photometry was as good as $5 \%$. Fluxes and equivalent widths of the emission lines were measured interactively using the integrate/line routine in MIDAS. The errors in the measurements were derived from repeated observations of the lines, and the errors of the fluxes and equivalent widths $W$ for different lines are given in Table 2 .

For all the galaxies with a strong underlying continuum emission (relative to the $\mathrm{H} \beta$ line emission, $W(\mathrm{H} \beta)<20 \AA$ ) we corrected the $\mathrm{H} \beta$ fluxes for underlying stellar absorption with an assumed constant equivalent width of $2 \AA$ (McCall et al. 1985). The $\mathrm{H} \beta$ fluxes were afterwards corrected for reddening due to dust in our own Galaxy and in the galaxy being observed. We used the reddening coefficient $c(\mathrm{H} \beta)$, derived from the observed $\mathrm{H} \alpha / \mathrm{H} \beta$ Balmer line ratios. From Eq. (7.6) of Osterbrock (1974), the intrinsic ratio $I(\mathrm{H} \alpha) / I(\mathrm{H} \beta)$ is related to the observed ratio via

$\frac{F(\mathrm{H} \alpha)}{F(\mathrm{H} \beta)}=\frac{I(\mathrm{H} \alpha)}{I(\mathrm{H} \beta)} \times 10^{-c}(\mathrm{H} \beta)[f(\mathrm{H} \alpha)-f(\mathrm{H} \beta)]$.

For the extinction in our Galaxy, $f(\mathrm{H} \alpha)-f(\mathrm{H} \beta)$ is the standard Galactic reddening law (Whitford 1958), with the extinction values taken from Burstein \& Heiles (1984). For the external extinction $f(\mathrm{H} \alpha)-f(\mathrm{H} \beta)$ is the reddening law given by Howarth (1983), which should be closer to the average reddening law for the dwarf galaxies in our sample than the standard galactic one. We assumed that the intrinsic Balmer-line ratios are equal to the case $B$ recombination values of Brocklehurst (1971) for an electron temperature of $10^{4} \mathrm{~K}$ and an electron density of $100 \mathrm{~cm}^{-3}$.

For all the objects considered in this paper we also obtained $B$ and $R$ frames. Most of the direct images were either obtained for the purpose of acquisition of the following spectroscopy or as a snap-shot survey to obtain total magnitudes. Dedicated deeper $(600 \mathrm{~s} B$ and $300 \mathrm{~s}$ $R$ ) images were observed for all isolated ELGs. The photometry of the whole sample is discussed in details in Vennik et al. (1999). Based on their photometric parameters (absolute magnitudes, diameters) and the morphological appearance on the CCD images, the galaxies were classified into the morphological classes proposed by Salzer et al. (1989a,b), namely Star Burst Nucleus Galaxies (SBN), Dwarf Amorphous Nuclear Starburst Galaxies (DANS), HII Hotspot Galaxies (HIIH), Dwarf HII Hotspot Galaxies (DHIIH), Sargent-Searle Objects (SS), Magellanic Irregular (Im), and Interacting Pairs (IP). Nevertheless, an independent check was done using the line ratios of the emission-lines and their location in the diagnostic diagrams, and there is an overall good agreement between the morphological and spectroscopic classification. 
Table 3.

\begin{tabular}{|c|c|c|c|c|c|c|c|c|c|c|}
\hline $\begin{array}{l}(1) \\
\text { Galaxy }\end{array}$ & $\begin{array}{l}(2) \\
M_{B}\end{array}$ & $\begin{array}{c}(3) \\
z\end{array}$ & $\begin{array}{r}(4) \\
c(\mathrm{H} \beta)\end{array}$ & $\begin{array}{r}(5) \\
\mathrm{H} \beta \\
W[\AA] \\
\end{array}$ & $\begin{array}{r}(6) \\
{[\mathrm{OIII}]} \\
W[\AA] \\
\end{array}$ & $\begin{array}{r}(7) \\
\mathrm{H} \alpha \\
W[\AA] \\
\end{array}$ & $\begin{array}{r}(8) \\
{[\mathrm{SII}]} \\
W[\AA] \\
\end{array}$ & $\begin{array}{c}(9) \\
F(\mathrm{H} \beta)_{\mathrm{obs}} \\
{\left[\mathrm{erg} \mathrm{s}^{-1} \mathrm{~cm}^{-2}\right]} \\
\end{array}$ & $\begin{array}{c}(10) \\
r_{\mathrm{HII}} \\
{\left[{ }^{\prime \prime}\right]} \\
\end{array}$ & $\begin{array}{l}(11) \\
\text { type }\end{array}$ \\
\hline HS $1222+3741$ & -17.67 & 0.0409 & 0.151 & 114 & 681 & 593 & 39 & $1.67 \mathrm{e}-14$ & 0.6 & $\mathrm{Im} / \mathrm{BCD}$ \\
\hline HS $1223+3938$ & -18.73 & 0.0360 & 0.474 & 15 & 91 & 101 & 14 & $6.88 \mathrm{e}-15$ & 1.7 & HIIH \\
\hline HS $1232+3846$ & -19.70 & 0.0528 & 0.254 & 11 & 14 & 75 & 23 & $4.18 \mathrm{e}-15$ & 2.0 & SBN \\
\hline HS $1232+3947$ & -17.16 & 0.0210 & 0.286 & 29 & 139 & 172 & 13 & $8.90 \mathrm{e}-15$ & 1.7 & DHIIH \\
\hline HS $1232+3612$ & -19.77 & 0.0425 & 0.411 & 18 & 85 & 136 & 25 & $1.27 \mathrm{e}-14$ & & IP \\
\hline HS $1236+3821$ & -17.06 & 0.0073 & 0.602 & 4 & 23 & 37 & 9 & $7.72 \mathrm{e}-15$ & 2.1 & DHIIH \\
\hline HS $1236+3937$ & -15.67 & 0.0184 & 0.081 & 112 & 506 & 521 & 56 & $5.72 \mathrm{e}-15$ & 0.8 & DHIIH/SS \\
\hline HS $1240+3755$ & -21.1 & 0.0860 & 0.969 & 3 & 6 & 64 & 14 & - & & IP/SBNpec \\
\hline HS $1244+3648$ & -18.82 & 0.0472 & 0.000 & 35 & 146 & 140 & 38 & $1.52 \mathrm{e}-14$ & 0.8 & HIIH \\
\hline HS $1256+3505$ & -18.75 & 0.0342 & 0.448 & 20 & 55 & 155 & 32 & $1.60 \mathrm{e}-14$ & 1.1 & DANS \\
\hline HS $1258+3438$ & -16.4 & 0.0248 & 0.380 & 36 & 209 & 189 & 15 & $4.39 \mathrm{e}-15$ & & DHIIH \\
\hline HS $1301+3312$ & -17.41 & 0.0371 & 0.391 & 31 & 125 & 177 & 40 & $5.90 \mathrm{e}-15$ & 0.8 & IP \\
\hline HS $1301+3325$ & -16.96 & 0.0246 & 0.573 & 11 & 51 & 73 & 8 & $3.71 \mathrm{e}-15$ & 1.3 & DHIIH \\
\hline HS $1301+3209$ & -17.06 & 0.0238 & 0.579 & 10 & 36 & 78 & 13 & $3.01 \mathrm{e}-15$ & 2.2 & HIIH \\
\hline HS $1304+3529$ & -17.38 & 0.0165 & 0.013 & 118 & 570 & 617 & 76 & $4.82 \mathrm{e}-14$ & 1.9 & IP \\
\hline HS $1306+3320$ & -18.32 & 0.0270 & 0.508 & 36 & 116 & 199 & 27 & $1.32 \mathrm{e}-14$ & 1.9 & HIIH \\
\hline HS $1308+3044$ & -17.92 & 0.0209 & 0.564 & 4 & 13 & 40 & 8 & $4.55 \mathrm{e}-15$ & 2.5 & DANS \\
\hline HS $1311+3628$ & -16.84 & 0.0031 & 0.160 & 301 & 1550 & 1834 & 139 & - & & DHIIH \\
\hline HS $1312+3847$ & -18.77 & 0.0515 & 0.037 & 74 & 337 & 392 & 58 & $1.53 \mathrm{e}-14$ & 1.4 & HIIH \\
\hline HS $1312+3508$ & -16.26 & 0.0035 & 0.164 & 254 & 1466 & 1463 & 179 & $1.02 \mathrm{e}-13$ & & DHIIH \\
\hline HS $1315+3132$ & -16.46 & 0.0315 & 0.476 & 36 & 136 & 175 & 28 & $4.14 \mathrm{e}-15$ & 0.8 & DHIIH \\
\hline HS $1318+3239$ & -17.22 & 0.0435 & 0.186 & 79 & 464 & 394 & 32 & $5.75 \mathrm{e}-15$ & 1.2 & IP \\
\hline HS $1319+3224$ & -15.3 & 0.0182 & 0.208 & 49 & 241 & 237 & 12 & - & 0.8 & SS/DHIIH \\
\hline HS $1325+3225$ & -17.9 & 0.0504 & 0.542 & 39 & 102 & 208 & 31 & - & & DHIIH/HIIH \\
\hline HS $1325+3255$ & -15.92 & 0.0263 & 0.146 & 74 & 412 & 340 & 14 & $4.38 \mathrm{e}-15$ & 0.8 & DHIIH/SS \\
\hline HS $1327+3126$ & -18.07 & 0.0568 & 0.192 & 91 & 514 & 484 & 42 & $1.07 \mathrm{e}-14$ & 0.6 & DHIIH \\
\hline HS $1328+3424$ & -17.79 & 0.0227 & 0.503 & 8 & 18 & 54 & 4 & $2.15 \mathrm{e}-15$ & & HIIH \\
\hline HS $1329+3703$ & -19.11 & 0.0557 & 0.214 & 7 & 9 & 54 & 15 & - & 1.9 & HIIH/DANS \\
\hline HS $1330+3651$ & -17.15 & 0.0167 & 0.147 & 72 & 387 & 371 & 45 & $2.62 \mathrm{e}-14$ & & DHIIH \\
\hline HS $1332+3426$ & -16.14 & 0.0220 & 0.444 & 35 & 167 & 195 & 17 & $4.27 \mathrm{e}-15$ & 1.4 & DHIIH \\
\hline HS $1334+3957$ & -15.32 & 0.0083 & 0.000 & 71 & 351 & 334 & 26 & - & 1.8 & DHIIH \\
\hline HS $1336+3114$ & -17.94 & 0.0158 & 0.000 & 5 & 11 & 29 & 14 & - & 3.9 & НIIH \\
\hline HS $1340+3307$ & -16.63 & 0.0158 & 0.539 & 20 & 108 & 128 & 23 & $9.73 \mathrm{e}-15$ & 1.6 & DHIIH \\
\hline HS $1341+3409$ & -16.35 & 0.0171 & 0.480 & 15 & 59 & 85 & 19 & $5.34 \mathrm{e}-15$ & 1.2 & DHIIH \\
\hline HS $1347+3811$ & -15.30 & 0.0103 & 0.243 & 64 & 363 & 290 & 33 & $8.62 \mathrm{e}-15$ & 4.9 & DHIIH \\
\hline HS $1349+3942$ & -15.16 & 0.0054 & 0.337 & 13 & 45 & 85 & 23 & $1.05 \mathrm{e}-14$ & 1.8 & DHIIH \\
\hline HS $1354+3634$ & -17.10 & 0.0167 & 0.513 & 19 & 50 & 123 & 26 & $1.12 \mathrm{e}-14$ & 1.2 & DANS \\
\hline HS $1354+3635$ & -17.81 & 0.0171 & 0.507 & 21 & 76 & 124 & 25 & $2.04 \mathrm{e}-14$ & 3.3 & HIIH \\
\hline HS $1402+3650$ & -18.81 & 0.0347 & 0.561 & 26 & 85 & 192 & 43 & $1.36 \mathrm{e}-14$ & 1.6 & HIIH \\
\hline HS $1410+3627$ & -18.11 & 0.0338 & 0.311 & 15 & 55 & 97 & 28 & $5.16 \mathrm{e}-15$ & & HIIH \\
\hline HS $1413+4402$ & -19.67 & 0.0698 & 0.607 & 10 & 5 & 99 & 13 & - & 1.3 & $\mathrm{SBN}$ \\
\hline HS $1416+3554$ & -16.94 & 0.0103 & 0.328 & 14 & 49 & 91 & 24 & $7.70 \mathrm{e}-15$ & 3.4 & HIIH/DHIIH \\
\hline HS $1420+3437$ & -16.75 & 0.0246 & 0.448 & 27 & 56 & 149 & 17 & $7.53 \mathrm{e}-15$ & 0.6 & DHIIH \\
\hline HS $1422+3325$ & -17.30 & 0.0341 & 0.337 & 20 & 50 & 119 & 30 & $4.69 \mathrm{e}-15$ & 0.8 & HIIH \\
\hline HS $1422+3339$ & -16.4 & 0.0114 & 0.350 & 17 & 58 & 95 & 20 & $1.12 \mathrm{e}-14$ & 1.8 & DHIIH \\
\hline HS $1424+3836$ & -16.06 & 0.0218 & 0.195 & 104 & 579 & 545 & 24 & $1.00 \mathrm{e}-14$ & 1.4 & DHIIH? \\
\hline HS $1425+3835$ & -17.80 & 0.0223 & 0.099 & 12 & 24 & 64 & 9 & - & 1.8 & HIIH \\
\hline HS $1429+4511$ & -17.40 & 0.0321 & 0.307 & 15 & 2 & 96 & 32 & - & 1.0 & DANS \\
\hline HS $1429+3154$ & -16.73 & 0.0117 & 0.143 & 34 & 144 & 162 & 26 & $2.06 \mathrm{e}-14$ & 2.6 & DHIIH \\
\hline HS $1438+3147$ & -17.88 & 0.0443 & 0.308 & 35 & 188 & 198 & 30 & $6.54 \mathrm{e}-15$ & 1.2 & DANS \\
\hline HS $1440+4302$ & -15.07 & 0.0085 & 0.336 & 44 & 269 & 238 & 31 & $1.13 \mathrm{e}-14$ & 1.2 & DHIIH/SS \\
\hline HS $1440+3120$ & -17.4 & 0.0525 & 0.221 & 152 & 925 & 845 & 62 & $9.60 \mathrm{e}-15$ & 0.7 & DHIIH \\
\hline HS $1440+3805$ & -18.80 & 0.0322 & 0.263 & 7 & 14 & 46 & 17 & - & 2.9 & HIIH \\
\hline
\end{tabular}


Table 3. continued

\begin{tabular}{|c|c|c|c|c|c|c|c|c|c|c|}
\hline $\begin{array}{l}\text { (1) } \\
\text { Galaxy }\end{array}$ & $\begin{array}{l}(2) \\
M_{B}\end{array}$ & $\begin{array}{c}(3) \\
z\end{array}$ & $\begin{array}{r}(4) \\
c(\mathrm{H} \beta)\end{array}$ & $\begin{array}{r}(5) \\
\mathrm{H} \beta \\
W[\AA] \\
\end{array}$ & $\begin{array}{r}(6) \\
{[\mathrm{OIII}]} \\
W[\AA] \\
\end{array}$ & $\begin{array}{r}(7) \\
\mathrm{H} \alpha \\
W[\AA] \\
\end{array}$ & $\begin{array}{r}(8) \\
{[\mathrm{SII}]} \\
W[\AA] \\
\end{array}$ & $\begin{array}{c}(9) \\
F(\mathrm{H} \beta)_{\mathrm{obs}} \\
{\left[\mathrm{erg} \mathrm{s}^{-1} \mathrm{~cm}^{-2}\right]}\end{array}$ & $\begin{array}{c}(10) \\
r_{\mathrm{HII}} \\
{\left[{ }^{\prime \prime}\right]} \\
\end{array}$ & $\begin{array}{l}\text { (11) } \\
\text { type }\end{array}$ \\
\hline HS $1442+4250$ & -14.73 & 0.0025 & 0.081 & 113 & 550 & 574 & 17 & $2.95 \mathrm{e}-14$ & & $\mathrm{SS}$ \\
\hline HS $1444+3114$ & -19.02 & 0.0297 & 0.413 & 26 & 60 & 174 & 38 & $2.78 \mathrm{e}-14$ & 1.7 & DANS \\
\hline HS $1502+4152$ & -16.66 & 0.0164 & 0.515 & 7 & 39 & 55 & 21 & $2.22 \mathrm{e}-15$ & & DHIIH \\
\hline HS $1507+3743$ & -17.58 & 0.0322 & 0.114 & 232 & 1374 & 1307 & 68 & $3.45 \mathrm{e}-14$ & 1.0 & DHIIH \\
\hline HS $1529+4512$ & -17.05 & 0.0231 & 0.222 & 16 & 99 & 102 & 12 & $2.95 \mathrm{e}-15$ & 1.7 & HIIH/DHIIH \\
\hline HS $1544+4736$ & -17.11 & 0.0195 & 0.025 & 32 & 154 & 162 & 30 & $1.00 \mathrm{e}-14$ & 2.6 & DHIIH \\
\hline HS $1546+4755$ & -17.78 & 0.0377 & 0.485 & 35 & 181 & 204 & 27 & $7.77 \mathrm{e}-15$ & 0.7 & DHIIH \\
\hline HS $1609+4827$ & -17.6 & 0.0096 & 0.381 & 12 & 40 & 86 & 20 & $1.91 \mathrm{e}-14$ & 2.5 & HIIH \\
\hline HS $1610+4539$ & -17.2 & 0.0196 & 0.480 & 26 & 106 & 152 & 24 & $1.41 \mathrm{e}-14$ & 1.3 & DHIIH/HIIH \\
\hline HS $1614+4709$ & -13.6 & 0.0026 & 0.100 & 140 & 880 & 915 & 55 & $6.62 \mathrm{e}-14$ & 1.6 & $\mathrm{SS}$ \\
\hline HS $1633+4703$ & -15.93 & 0.0086 & 0.364 & 15 & 55 & 83 & 19 & $1.34 \mathrm{e}-14$ & 1.1 & DHIIH \\
\hline HS $1640+5136$ & -19.59 & 0.0308 & 0.443 & 21 & 55 & 167 & 34 & $2.42 \mathrm{e}-14$ & 2.0 & $\mathrm{SBN} / \mathrm{HIIH}$ \\
\hline HS $1641+5053$ & -19.05 & 0.0292 & 0.574 & 15 & 65 & 110 & 21 & $2.11 \mathrm{e}-14$ & & HIIH \\
\hline HS $1645+5155$ & -19.11 & 0.0286 & 0.330 & 47 & 191 & 265 & 45 & $1.53 \mathrm{e}-14$ & 5.5 & HIIH \\
\hline HS $1657+5735$ & -20.60 & 0.0505 & 0.455 & 43 & 167 & 253 & 46 & $5.43 \mathrm{e}-14$ & 1.2 & $\mathrm{SBN}$ \\
\hline HS $1723+5631 \mathrm{~A}$ & -18.70 & 0.0286 & 0.341 & 23 & 100 & 121 & 20 & $1.13 \mathrm{e}-14$ & & IP \\
\hline HS $1723+5631 B$ & -18.70 & 0.0286 & 0.337 & 32 & 140 & 188 & 32 & $1.16 \mathrm{e}-14$ & & IP \\
\hline HS $1728+5655$ & -16.38 & 0.0160 & 0.094 & 106 & 514 & 522 & 36 & $2.39 \mathrm{e}-14$ & 0.9 & DHIIH \\
\hline
\end{tabular}

\section{The Catalogue}

The Catalogue is presented in Table 3 through Table 6 . Tables 3 and 4 present the spectroscopic parameters of all the galaxies for which a reliable internal extinction correction was done, and which account for the main body of the Catalogue. The data are arranged in Table 3 as follows:

- Column 1: The galaxy name according to Popescu et al. $(1996,1998)$.

- Column 2: The absolute blue magnitudes $\left(M_{B}\right)$ as listed in Vennik et al. (1999).

- Column 3: The heliocentric redshift.

- Column 4: The absorption coefficient $c(\mathrm{H} \beta)$.

- Columns 5, 6, 7, 8: The equivalent widths $(W)$ of the $\mathrm{H} \beta,[\mathrm{OIII}] \lambda 5007, \mathrm{H} \alpha$ and $[\mathrm{SII}] \lambda \lambda 6717,6731$ emission lines in $\AA$.

- Column 9: The observed $\mathrm{H} \beta$ fluxes in $\mathrm{erg} \mathrm{s}^{-1} \mathrm{~cm}^{-2}$, corrected for absorption.

- Column 10: Seeing corrected approximate angular size of the star-forming regions $r_{\mathrm{HII}}$, as measured in the $B$ band by Vennik et al. (2000).

- Column 11: The morphological type.

Table 4 contains the corrected line ratios (normalised to $\mathrm{H} \beta=100$ ) of the emission lines detected in the spectra of the galaxies listed in Table 3 . The header of the table contains the identification of the emission line while the line below gives the corresponding restframe wavelength. The [SII] $\lambda \lambda 6724,6731$ and the [OII] $\lambda \lambda 7320,7330$ are not deblended, and therefore the line ratios refer to their blend.
Table 5 lists the galaxies for which it was not possible to deblend $\mathrm{H} \alpha$ from $[\mathrm{NII}] \lambda \lambda 6563,6584$, and therefore the line ratios were not corrected for internal extinction. The line ratio of $\mathrm{H} \alpha$ corresponds to the blend with the [NII] lines. Finally, Table 6 lists those galaxies for which the $\mathrm{H} \beta$ emission line was too noisy to allow an accurate determination of the internal extinction, and again only the uncorrected line ratios are listed. Here, the line ratios in Col. 5 through 8 are given in units of $\mathrm{H} \alpha=100$.

In 8 cases it was possible to disentangle the contribution of different HII regions in the galaxy. Then the spectrum of each region was extracted separately and the fluxes measured for each individual region. In Tables 3-6 only the line ratios corresponding to the brightest HII region are given, but the $\mathrm{H} \beta$ fluxes are the total fluxes for the galaxy, summed over all the HII regions. In Table 7 we list the 8 galaxies with detected HII regions together with their observed $\mathrm{H} \beta$ fluxes. These fluxes are not corrected for absorption. The HII regions are given in order of their location along the slit, from South to North.

\section{Wolf-Rayet galaxies}

Some of our galaxies show the broad HeII $\lambda 4686$ feature, which is a direct signature from Wolf-Rayet (WR) stars. For low resolution spectroscopy (as in the case of our spectra) this line is usually seen as a WR bump, a blend of HeII and other broad stellar emission lines from CIII, NIII 


\begin{tabular}{|c|c|c|c|c|c|c|c|c|c|c|c|c|c|c|c|c|c|}
\hline $\begin{array}{l}\text { (1) } \\
\text { Galaxy }\end{array}$ & $\begin{array}{r}(2) \\
{[\mathrm{OII}]} \\
3727\end{array}$ & $\begin{array}{r}(3) \\
{[\mathrm{NeIII}]} \\
3869\end{array}$ & $\begin{array}{r}(4) \\
\mathrm{H} \delta \\
4102\end{array}$ & $\begin{array}{r}(5) \\
\mathrm{H} \gamma \\
4340\end{array}$ & $\begin{array}{r}(6) \\
{[\mathrm{OIII}]} \\
4363\end{array}$ & $\begin{array}{r}(7) \\
{[\mathrm{OIII}]} \\
4959\end{array}$ & $\begin{array}{r}(8) \\
{[\mathrm{OIII}]} \\
5007\end{array}$ & $\begin{array}{r}(9) \\
\mathrm{HeI} \\
5876\end{array}$ & $\begin{array}{r}(10) \\
{[\mathrm{OI}]} \\
6300\end{array}$ & $\begin{array}{r}(11) \\
{[\mathrm{OI}]} \\
6364\end{array}$ & $\begin{array}{c}(12) \\
{[\mathrm{NII}]} \\
6584\end{array}$ & $\begin{array}{r}(13) \\
\mathrm{HeI} \\
6678\end{array}$ & $\begin{array}{l}(14) \\
{[\mathrm{SII}]} \\
6717 \\
6731 \\
\end{array}$ & $\begin{array}{r}(15) \\
\text { HeI } \\
7065\end{array}$ & $\begin{array}{r}(16) \\
{[\mathrm{AIII}]} \\
7136\end{array}$ & $\begin{array}{r}(17) \\
{[\mathrm{OII}]} \\
7320 \\
7330 \\
\end{array}$ & $\begin{array}{r}(18) \\
\text { [AIII] } \\
7751\end{array}$ \\
\hline HS $1222+3741$ & 168 & 68 & 22 & 45 & 15 & 185 & 556 & 10 & 4 & - & - & - & 16 & - & 5 & - & - \\
\hline HS $1223+3938$ & 379 & 75 & - & 36 & - & 177 & 509 & 17 & - & - & - & - & 38 & - & 3 & - & - \\
\hline HS $1232+3846$ & 334 & - & - & 32 & - & 38 & 103 & - & - & - & 68 & - & 109 & - & - & - & - \\
\hline HS $1232+3947$ & 256 & - & - & 34 & - & 140 & 427 & 14 & 16 & - & 5 & 9 & 21 & - & - & - & - \\
\hline HS $1232+3612$ & 428 & 69 & - & 43 & - & 132 & 399 & 9 & 13 & - & 27 & 7 & 56 & - & 4 & - & - \\
\hline HS $1236+3821$ & 849 & - & - & - & - & 127 & 380 & 11 & 8 & - & 19 & - & 73 & - & - & - & - \\
\hline HS $1236+3937$ & 116 & 78 & 27 & 51 & 14 & 152 & 445 & 13 & - & - & - & - & 26 & - & - & - & - \\
\hline HS $1240+3755$ & 659 & - & - & - & - & 76 & 94 & - & - & - & 179 & - & 96 & - & - & - & - \\
\hline HS $1244+3648$ & 292 & 55 & - & 41 & - & 135 & 406 & 15 & 16 & 7 & - & - & 72 & - & 5 & - & - \\
\hline HS $1256+3505$ & 467 & - & - & 60 & - & 87 & 251 & 15 & 7 & - & 63 & - & 73 & - & 9 & - & - \\
\hline HS $1258+3438$ & 222 & 43 & 23 & 48 & 2 & 193 & 534 & 10 & - & - & - & - & 22 & - & - & - & - \\
\hline HS $1301+3312$ & 439 & - & - & 44 & - & 131 & 393 & 9 & 17 & - & - & - & 58 & - & - & - & - \\
\hline HS $1301+3325$ & 508 & - & - & - & - & 125 & 350 & - & - & - & - & - & 28 & - & - & - & - \\
\hline HS $1301+3209$ & 912 & - & - & - & - & 99 & 287 & - & 19 & - & 10 & - & 52 & - & - & - & - \\
\hline HS $1304+3529$ & 185 & 55 & 24 & 46 & 10 & 152 & 451 & 10 & 9 & - & 10 & 1 & 33 & - & 6 & - & - \\
\hline HS $1306+3320$ & 354 & 53 & - & 50 & - & 108 & 320 & 11 & 7 & - & - & - & 37 & - & 8 & - & - \\
\hline HS $1308+3044$ & 577 & - & - & - & - & 70 & 198 & - & - & - & 59 & - & 68 & - & - & - & - \\
\hline HS $1311+3628$ & 216 & 29 & 30 & 54 & - & 176 & 492 & 11 & 4 & - & 13 & 3 & 28 & - & 9 & 3 & - \\
\hline HS $1312+3847$ & 242 & 64 & 18 & 46 & 5 & 153 & 445 & 18 & 6 & - & 10 & - & 47 & - & 7 & - & - \\
\hline HS $1312+3508$ & 261 & 64 & 29 & 53 & 7 & 170 & 492 & 12 & 5 & 3 & 11 & 5 & 30 & 4 & 11 & 5 & 4 \\
\hline HS $1315+3132$ & 410 & 20 & 24 & 50 & 5 & 123 & 356 & 11 & 10 & - & - & - & 42 & - & 8 & - & - \\
\hline HS $1318+3239$ & 156 & 63 & 27 & 44 & 12 & 198 & 577 & 13 & - & - & - & 3 & 23 & - & 6 & - & - \\
\hline HS $1319+3224$ & 124 & 63 & 26 & 48 & 13 & 163 & 503 & 10 & - & - & - & - & 14 & - & - & - & - \\
\hline HS $1325+3225$ & 353 & - & - & 44 & - & 84 & 250 & 10 & - & - & - & 3 & 40 & - & - & - & - \\
\hline HS $1325+3255$ & 126 & 57 & 22 & 49 & 8 & 187 & 548 & 9 & 6 & - & - & - & 12 & - & - & - & - \\
\hline HS $1327+3126$ & 199 & 56 & 26 & 46 & 7 & 182 & 536 & 12 & 5 & 4 & - & 3 & 25 & 3 & 5 & 3 & 2 \\
\hline HS $1328+3424$ & 434 & - & - & - & - & 84 & 183 & - & - & - & 34 & - & 25 & - & - & - & - \\
\hline HS $1329+3703$ & 366 & - & - & - & - & 35 & 102 & - & - & - & 85 & - & 111 & - & - & - & - \\
\hline HS $1330+3651$ & 186 & 48 & 25 & 43 & 9 & 164 & 490 & 15 & 8 & - & 9 & - & 35 & - & 4 & - & - \\
\hline HS $1332+3426$ & 359 & 74 & - & 53 & - & 162 & 455 & 14 & 11 & - & - & - & 23 & - & 6 & 2 & - \\
\hline HS $1334+3957$ & 288 & 48 & 33 & 55 & - & 178 & 519 & 17 & - & - & 5 & - & 24 & - & 7 & - & - \\
\hline
\end{tabular}




\begin{tabular}{|c|c|c|c|c|c|c|c|c|c|c|c|c|c|c|c|c|c|}
\hline $\begin{array}{l}\text { (1) } \\
\text { Galaxy }\end{array}$ & $\begin{array}{r}(2) \\
{[\mathrm{OII}]} \\
3727\end{array}$ & $\begin{array}{r}(3) \\
{[\mathrm{NeIII}]} \\
3869\end{array}$ & $\begin{array}{r}(4) \\
\mathrm{H} \delta \\
4102\end{array}$ & $\begin{array}{r}(5) \\
\mathrm{H} \gamma \\
4340\end{array}$ & $\begin{array}{r}(6) \\
{[\mathrm{OIII}]} \\
4363\end{array}$ & $\begin{array}{r}(7) \\
{[\mathrm{OIII}]} \\
4959\end{array}$ & $\begin{array}{r}(8) \\
{[\mathrm{OIII}]} \\
5007\end{array}$ & $\begin{array}{r}(9) \\
\text { HeI } \\
5876\end{array}$ & $\begin{array}{r}(10) \\
{[\mathrm{OI}]} \\
6300\end{array}$ & $\begin{array}{r}(11) \\
{[\mathrm{OI}]} \\
6364\end{array}$ & $\begin{array}{r}(12) \\
{[\mathrm{NII}]} \\
6584\end{array}$ & $\begin{array}{r}(13) \\
\mathrm{HeI} \\
6678\end{array}$ & $\begin{array}{l}(14) \\
{[\mathrm{SII}]} \\
6724 \\
6731 \\
\end{array}$ & $\begin{array}{r}(15) \\
\text { HeI } \\
7065\end{array}$ & $\begin{array}{r}(16) \\
{[\mathrm{AIII}]} \\
7136\end{array}$ & $\begin{array}{r}(17) \\
{[\mathrm{OII}]} \\
7320 \\
7330 \\
\end{array}$ & $\begin{array}{r}(18) \\
{[\mathrm{AIII}]} \\
7751\end{array}$ \\
\hline HS $1336+3114$ & 278 & - & - & - & - & 79 & 172 & - & - & - & 51 & - & 163 & - & - & - & - \\
\hline HS $1340+3307$ & 503 & 153 & - & 66 & - & 174 & 485 & 16 & - & - & 7 & - & 49 & - & - & - & - \\
\hline HS $1341+3409$ & 573 & 88 & - & - & - & 126 & 325 & 18 & - & - & - & - & 60 & - & - & - & - \\
\hline HS $1347+3811$ & 229 & 90 & 28 & 54 & 7 & 189 & 546 & 13 & 8 & - & - & - & 33 & 3 & 8 & - & - \\
\hline HS $1349+3942$ & 556 & - & - & 53 & - & 106 & 286 & 13 & 12 & - & 26 & - & 82 & - & - & - & - \\
\hline HS $1354+3634$ & 467 & - & - & 48 & - & 91 & 255 & 10 & 18 & - & 44 & - & 71 & - & - & - & - \\
\hline HS $1354+3635$ & 468 & - & - & 48 & - & 118 & 339 & 10 & 7 & - & 28 & 3 & 61 & - & 4 & - & - \\
\hline HS $1402+3650$ & 483 & 85 & - & 49 & - & 96 & 294 & 12 & 13 & - & 20 & 4 & 63 & - & - & - & - \\
\hline HS $1410+3627$ & 601 & - & - & 46 & - & 109 & 305 & 14 & 14 & - & 14 & - & 81 & - & - & - & - \\
\hline HS $1413+4402$ & 214 & - & - & - & - & 26 & 45 & - & - & - & 150 & - & 62 & - & - & - & - \\
\hline HS $1416+3554$ & 593 & 87 & - & - & - & 99 & 284 & - & - & - & 18 & - & 77 & - & - & - & - \\
\hline HS $1420+3437$ & 484 & - & - & 49 & - & 79 & 196 & 11 & - & - & 22 & - & 34 & - & - & - & - \\
\hline HS $1422+3325$ & 479 & - & - & 50 & - & 90 & 245 & 9 & - & - & 59 & - & 86 & - & - & - & - \\
\hline HS $1422+3339$ & 466 & - & - & 40 & - & 105 & 289 & - & - & - & 21 & - & 65 & - & - & - & - \\
\hline HS $1424+3836$ & 115 & 36 & 26 & 53 & 7 & 181 & 552 & 12 & 4 & - & - & - & 12 & - & 3 & - & - \\
\hline HS $1425+3835$ & 586 & - & - & - & - & 59 & 200 & - & - & - & 40 & - & 51 & - & - & - & - \\
\hline HS $1429+4511$ & 205 & - & - & - & - & 11 & 51 & - & - & - & 82 & - & 112 & - & - & - & 0 \\
\hline HS $1429+3154$ & 392 & - & - & 46 & - & 142 & 419 & 6 & 6 & - & 19 & - & 49 & - & 7 & - & - \\
\hline HS $1438+3147$ & 335 & 71 & - & 43 & - & 174 & 507 & 14 & 8 & - & 7 & - & 42 & - & - & - & - \\
\hline HS $1440+4302$ & 530 & 94 & 26 & 60 & 14 & 191 & 558 & 10 & 6 & - & 11 & - & 36 & - & 7 & - & - \\
\hline HS $1440+3120$ & 156 & 59 & 30 & 44 & 11 & 211 & 613 & 13 & 5 & - & - & 3 & 20 & - & 4 & - & - \\
\hline HS $1440+3805$ & 397 & - & - & - & - & 78 & 159 & 13 & 23 & - & 50 & - & 118 & - & - & - & - \\
\hline HS $1442+4250$ & 97 & 50 & 22 & 38 & 11 & 165 & 475 & 12 & - & - & - & 2 & 8 & - & 4 & - & - \\
\hline HS $1444+3114$ & 492 & 30 & - & 50 & - & 75 & 220 & 9 & 9 & 5 & 43 & - & 71 & - & 6 & - & - \\
\hline HS $1502+4152$ & - & - & - & - & - & 153 & 420 & - & - & - & 22 & - & 108 & - & - & - & - \\
\hline HS $1507+3743$ & 87 & 66 & 29 & 52 & 12 & 217 & 658 & 10 & 3 & - & - & 2 & 13 & 4 & 4 & 4 & - \\
\hline HS $1529+4512$ & 465 & 76 & - & 46 & - & 173 & 502 & - & - & - & 16 & - & 34 & - & - & - & 0 \\
\hline HS $1544+4736$ & 104 & - & - & 62 & - & 147 & 456 & 15 & 7 & - & 14 & - & 52 & - & 7 & - & - \\
\hline HS $1546+4755$ & 397 & 73 & 40 & 52 & - & 166 & 494 & 10 & 7 & - & 6 & - & 37 & - & - & - & - \\
\hline
\end{tabular}




\begin{tabular}{|c|c|c|c|c|c|c|c|c|c|c|c|c|c|c|c|c|c|}
\hline (1) & $(2)$ & (3) & (4) & (5) & (6) & $(7)$ & (8) & (9) & (10) & (11) & $(12)$ & (13) & (14) & (15) & (16) & (17) & (18) \\
\hline \multirow[t]{2}{*}{ Galaxy } & [OII] & [NeIII] & $\mathrm{H} \delta$ & $\mathrm{H} \gamma$ & [OIII] & [OIII] & [OIII] & $\mathrm{HeI}$ & {$[\mathrm{OI}]$} & {$[\mathrm{OI}]$} & {$[\mathrm{NII}]$} & $\mathrm{HeI}$ & {$[\mathrm{SII}]$} & $\mathrm{HeI}$ & [AIII] & [OII] & [AIII] \\
\hline & 3727 & 3869 & 4102 & 4340 & 4363 & 4959 & 5007 & 5876 & 6300 & 6364 & 6584 & 6678 & $\begin{array}{l}6724 \\
6731\end{array}$ & 7065 & 7136 & $\begin{array}{l}7320 \\
7330\end{array}$ & 7751 \\
\hline
\end{tabular}

\begin{tabular}{|c|c|c|c|c|c|c|c|c|c|c|c|c|c|c|c|c|c|}
\hline HS $1609+4827$ & 551 & - & - & 38 & - & 91 & 261 & 8 & 15 & - & - & - & 63 & - & - & - & - \\
\hline HS $1610+4539$ & 390 & - & 31 & 47 & 9 & 136 & 374 & 10 & 10 & - & - & 4 & 42 & - & 4 & - & - \\
\hline HS $1614+4709$ & 79 & 54 & 27 & 50 & 6 & 200 & 590 & 10 & 4 & - & - & - & 18 & - & 5 & - & - \\
\hline HS $1633+4703$ & 596 & 86 & - & 48 & - & 116 & 309 & 15 & 10 & - & 19 & 9 & 67 & - & 7 & - & - \\
\hline HS $1640+5136$ & 520 & 22 & - & 56 & - & 84 & 243 & 15 & 11 & 4 & 98 & - & 79 & - & 5 & - & - \\
\hline HS $1641+5053$ & 582 & 94 & - & 33 & - & 121 & 339 & 11 & 11 & - & - & - & 50 & - & - & - & - \\
\hline HS $1645+5155$ & 349 & - & 24 & 45 & 9 & 129 & 387 & 22 & 7 & - & 12 & - & 46 & - & - & - & - \\
\hline HS $1657+5735$ & 349 & 39 & 22 & 47 & 3 & 117 & 355 & 10 & 7 & 1 & - & 2 & 47 & - & 3 & - & 2 \\
\hline HS $1723+5631 \mathrm{~A}$ & 425 & - & - & 61 & - & 140 & 412 & 15 & 14 & 6 & 23 & - & 49 & - & - & - & - \\
\hline HS 1723+5631B & 352 & - & - & 54 & - & 144 & 419 & 13 & 9 & - & 23 & - & 50 & - & - & - & - \\
\hline HS $1728+5655$ & 211 & 75 & 26 & 47 & 3 & 172 & 500 & 12 & 5 & 2 & 25 & 2 & 23 & - & 7 & 3 & - \\
\hline
\end{tabular}

\begin{tabular}{|c|c|c|c|c|c|c|c|c|c|c|c|c|c|c|c|c|c|c|}
\hline $\begin{array}{l}\text { (1) } \\
\text { Galaxy }\end{array}$ & $\begin{array}{l}(2) \\
M_{B}\end{array}$ & $\begin{array}{c}(3) \\
z\end{array}$ & $\begin{array}{c}(4) \\
\mathrm{H} \beta \\
W \\
{[\AA]} \\
{[}\end{array}$ & $\begin{array}{r}(5) \\
{[\mathrm{OIII}]} \\
W \\
{[\AA]} \\
\end{array}$ & $\begin{array}{c}(6) \\
\mathrm{H} \alpha \\
W \\
{[\AA]} \\
{[}\end{array}$ & $\begin{array}{r}(7) \\
{[\mathrm{SII}]} \\
W \\
{[\AA]} \\
\end{array}$ & $\begin{array}{c}(8) \\
F(\mathrm{H} \beta)_{\mathrm{obs}} \\
{\left[\mathrm{erg} \mathrm{s}^{-1} \mathrm{~cm}^{-2}\right]}\end{array}$ & $\begin{array}{r}(9) \\
{[\mathrm{OII}]} \\
3727\end{array}$ & $\begin{array}{r}(10) \\
\mathrm{H} \gamma \\
4340\end{array}$ & $\begin{array}{r}(11) \\
{[\text { OIII] }} \\
4959\end{array}$ & $\begin{array}{r}(12) \\
{[\mathrm{OIII}]} \\
5007\end{array}$ & $\begin{array}{c}(13) \\
\mathrm{HeI} \\
5876\end{array}$ & $\begin{array}{c}(14) \\
{[\mathrm{OI}]} \\
6300\end{array}$ & $\begin{array}{l}(15) \\
\mathrm{H} \alpha^{*} \\
6563 \\
6584 \\
\end{array}$ & $\begin{array}{r}(16) \\
\mathrm{HeI} \\
6678\end{array}$ & $\begin{array}{l}(17) \\
{[\mathrm{SII}]} \\
6724 \\
6731 \\
\end{array}$ & $\begin{array}{r}(18) \\
r_{\mathrm{HII}} \\
{\left[{ }^{\prime \prime}\right]} \\
\end{array}$ & $\begin{array}{l}\text { (19) } \\
\text { type }\end{array}$ \\
\hline HS $1255+3506$ & -16.61 & 0.0155 & 10 & 31 & 66 & 19 & $4.70 \mathrm{e}-15$ & 381 & - & 86 & 258 & - & 24 & 457 & - & 124 & 1.7 & DHIIH? \\
\hline HS $1318+3406$ & -18.65 & 0.0352 & 8 & 17 & 69 & 21 & $5.09 \mathrm{e}-15$ & 414 & - & 74 & 178 & - & - & 526 & - & 152 & & HIIH? \\
\hline HS $1327+3412$ & - & 0.2510 & 11 & 7 & 111 & 11 & - & 87 & - & 13 & 52 & - & - & 376 & - & 37 & 0.7 & - \\
\hline HS $1333+3149$ & -18.61 & 0.0248 & 8 & 27 & 66 & 11 & $8.86 \mathrm{e}-15$ & 387 & - & 101 & 267 & - & - & 470 & - & 77 & 2.3 & HIIH \\
\hline HS $1340+3207$ & -18.35 & 0.0365 & 18 & 105 & 127 & 23 & $6.92 \mathrm{e}-15$ & 390 & 16 & 186 & 555 & 21 & - & 426 & - & 73 & 1.7 & DANS \\
\hline HS $1429+3154^{*}$ & -16.73 & 0.0117 & 34 & 144 & 162 & 26 & $2.06 \mathrm{e}-14$ & 347 & 39 & 143 & 424 & 6 & 7 & 344 & - & 55 & 2.6 & DHIIH \\
\hline HS $1442+4332$ & -20.05 & 0.0811 & 5 & 9 & 65 & 15 & $2.55 \mathrm{e}-15$ & 361 & - & 57 & 116 & 15 & 43 & 616 & 14 & 126 & 1.5 & SBN? \\
\hline HS $1543+4525$ & -19.17 & 0.0389 & 10 & 15 & 92 & 27 & $6.90 \mathrm{e}-15$ & 388 & - & 38 & 123 & 15 & 14 & 573 & 8 & 164 & 2.2 & DANS? \\
\hline HS $1627+5239^{*}$ & -17.88 & 0.0288 & 14 & 37 & 95 & 16 & $7.61 \mathrm{e}-15$ & 286 & - & 73 & 224 & 12 & - & 422 & - & 70 & 1.22 & HIIH? \\
\hline HS $1657+5033$ & -17.43 & 0.0102 & 7 & 29 & 60 & 14 & $1.19 \mathrm{e}-14$ & 338 & - & 103 & 323 & 10 & - & 559 & 6 & 124 & 1.88 & HIIH \\
\hline
\end{tabular}

The galaxies HS $1429+3154$ and HS $1627+5239$ have detected [AIII] $\lambda 7136$, with the line ratios relative to H $\beta$ of 8 and 15 , respectively. 
and NV, but also of narrow nebular emission lines including HeII $\lambda 4686$. The galaxies with detected WR features are listed in Table 8 with the flag D (detection), while a few more candidates for WR galaxies are also listed in Table 8, with the flag $\mathrm{C}$ (candidates). For some of the galaxies with detected WR features we also list the line ratios (corrected for internal extinction) relative to $\mathrm{H} \beta=$ 100. The first galaxy from the table, HS 0915+5540, does not belong to the spectrophotometric catalogue presented in this paper, but to the larger catalogue of emission-line galaxies from which the present catalogue was selected (Popescu et al. 1996).

In total we detected 6 new WR galaxies and 3 candidates for WR galaxies. This represent $5.5 \%$ from our spectrophotometrical catalogue, or $9 \%$, if we include the candidates, too. Such a detection rate is somewhat lower than the detection rate of $10 \%$ obtained by Masegosa et al. (1991) for a systematic search for broad WR bump in the HII galaxies. However our spectral resolution is very low and it is therefore not optimised for this kind of search, and these results came only serendipitously.

The total number of known WR galaxies and extragalactic HII regions showing broad HeII $\lambda 4686$ emission is 139 (from the recent compilation of Schaerer et al. 1999). These objects are found among a large variety of morphological types, from BCDs and Im galaxies to massive spirals and luminous IRAS galaxies, and even in Seyfert 2 and LINERSs (Osterbrock \& Cohen 1982; Kunth \& Contini 1998). Extending the sample of WR galaxies is very useful in constraining the evolution of massive stars and the parameters of the upper part of the IMF. Also, as discussed by Schaerer et al. (1999), WR galaxies represent useful templates of young starburst galaxies, which may be used to explain the properties of the distant starforming galaxies.

Among the galaxies with detected WR features we found one SBN galaxy (HS 1657+5735), a DANS galaxy (HS 0915+5540), three DHIIH galaxies (HS 1312+3508,

HS $1424+3836$, HS $1507+3743)$, and a interacting pair, IP (HS 1304+3529). The candidates for WR features are SS, HIIH and DHIIH galaxies. Thus it is probably reasonable to conclude that WR phenomenon is spread over all morphological subtypes of BCDs.

\section{Candidates for very low metallicities}

There has been a long debate on whether the BCDs are truly young dwarf galaxies undergoing their first burst of star formation or whether the present burst occurs within an older galaxy. The low abundances found in these galaxies make them good candidates for the least chemically evolved galaxies. But the detection of an extended faint stellar underlying component in the majority of BCDs (Loose \& Thuan 1986; Kunth et al. 1988; Telles et al. 1997; Vennik et al. 1999) supports the idea that they are not truly primordial galaxies, but older LSB dwarf galaxies undergoing transient periods of star formation. A clear demonstration of this two - component structure was recently presented by Schulte-Ladbeck et al. (1998, 1999) for the very nearby BCD VII Zw 403 (UGC 6456), based on HST photometry of its individual stars down to the red giant branch stars. Even for I Zw 18, the lowest metallicity known galaxy, Garnett et al. (1997) found C/O to be much larger than the mean value of other metal-deficient galaxies. This was interpreted as a proof that I Zw 18 has experienced carbon-enriching episodes of star formation in the past, and is therefore not a young galaxy. Recently Aloisi et al. (1999) used synthetic colour-magnitude diagrams to investigate deep HST data for I Zw 18, and they found again that the present burst is not the first one to occur in this galaxy.

However, Izotov \& Thuan (1999) argued that the extreme low metallicity BCD SBS 0335-052 is a good example for a young galaxy; the HST $V$ and $I$ imaging of this galaxy (Thuan et al. 1997) showed extremely blue colours, not only in the region of current star formation but also in the extended low surface brightness underlying component. Thuan \& Izotov (1997) also argued that the large HI cloud associated with this BCG (Pustilnik et al. 1999 ) is made of pristine gas, unpolluted by metals. Based on abundance measurements, Izotov \& Thuan (1999) suggested that in fact all galaxies with $12+\log (\mathrm{O} / \mathrm{H}) \leq 7.6$ are young, with ages not exceeding $40 \mathrm{Myr}$, while those with $7.6<12+\log (\mathrm{O} / \mathrm{H})<8.2$ are not older than $1-2$ Gyr. Furthermore, Lynds et al. (1998) discussed the possibility that VII Zw 403 (UGC 6456) is not young (as also found by Schulte-Ladbeck et al. 1998, 1999), but could still be considerably younger than a Hubble time.

While the controversy on the age of the BCDs reflects our current knowledge of galaxy formation and evolution (Schulte-Ladbeck et al. 1999), more work on both statistical samples of very low-metallicity BCDs as well as HST imaging of nearby BCDs could give clues relevant to this debate. In this section we give a list of candidates for very-low metallicity BCDs, which were found in the spectroscopic analyses of our sample. Nevertheless, highresolution spectroscopy is needed to confirm the metallicity that we assign for each object.

We derived the metallicities for our sample using both the models of Dopita \& Evans (1986) as well as a five level atom program and the ionisation correction method of Mathis \& Rosa (1991), the latter for the few objects for which the [OIII] $\lambda 4363$ line was detected in the spectrum. The distribution of $12+\log (\mathrm{O} / \mathrm{H})$ (Fig. 1) has a maximum around 8.5, with a long tail towards low and very low metallicities. All the galaxies with metallicities less than 8.0 were considered candidates for very low metallicity objects and are listed in Table 9. The value of the metallicity we assign should be taken with caution, and only as a preliminary result. As a simple consistency check, 
Table 6.

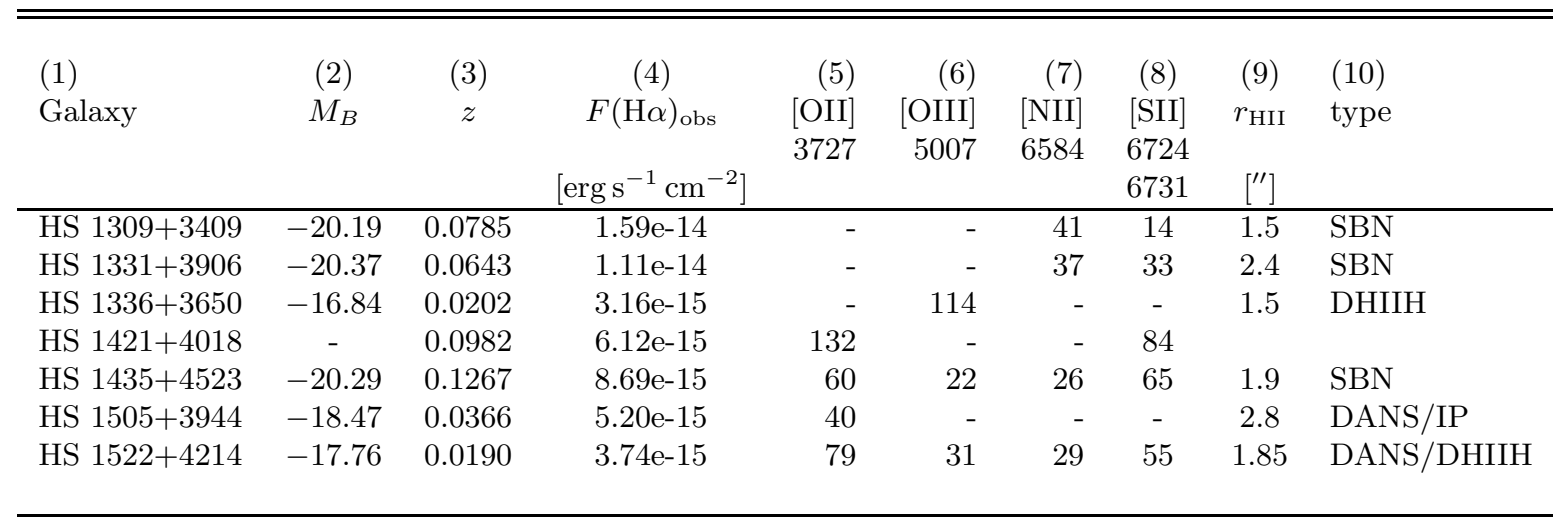

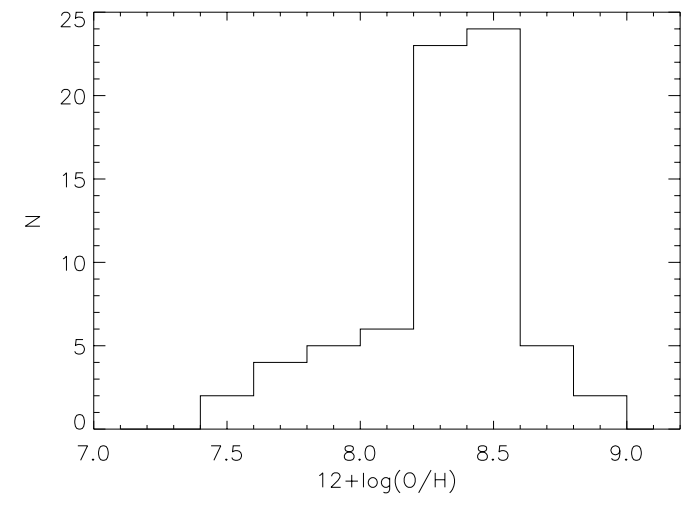

Fig. 1. The distribution of the oxygen abundances $12+\log (\mathrm{O} / \mathrm{H})$

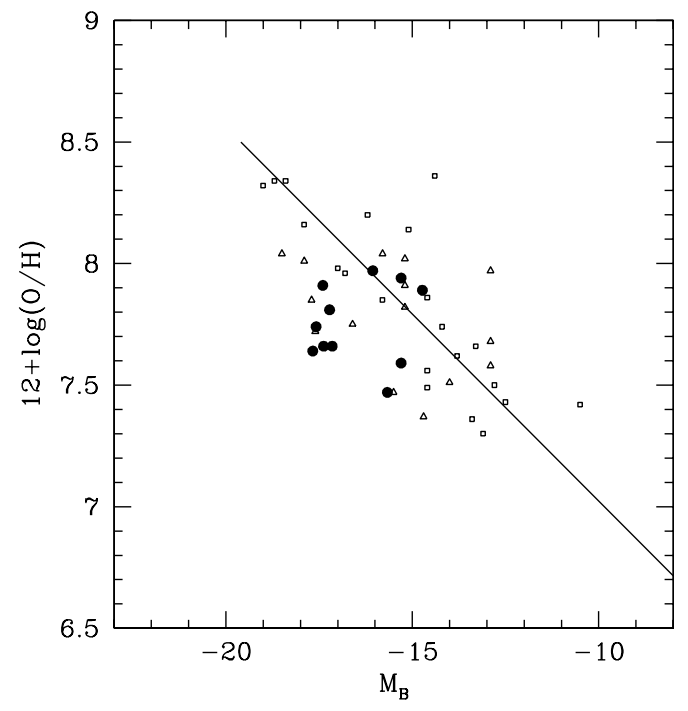

Fig. 2. The oxygen abundances $(12+\log (\mathrm{O} / \mathrm{H}))$ - absolute magnitude relation as derived by Skillman et al. (1989) for the local dwarf irregular galaxies (open squares). Some selected BCDs from Thuan et al. (1997) are also plotted (open triangles) together with our low-abundance candidates (filled dots)
Table 7.

\begin{tabular}{ccccc}
\hline \hline \multirow{2}{*}{ Galaxy } & \multicolumn{4}{c}{$F(\mathrm{H} \beta)_{\text {obs }}\left[\mathrm{erg} \mathrm{s}^{-1} \mathrm{~cm}^{-2}\right]$} \\
& HII1 & HII2 & HII3 & HII4 \\
\hline & & & & \\
HS 1232+3846 & $4.25 \mathrm{e}-16$ & $2.33 \mathrm{e}-15$ & $4.19 \mathrm{e}-16$ & $3.62 \mathrm{e}-16$ \\
HS 1236+3821 & $5.01 \mathrm{e}-15$ & $1.90 \mathrm{e}-16$ & & \\
HS 1244+3648 & $1.25 \mathrm{e}-15$ & $1.39 \mathrm{e}-14$ & & \\
HS 1304+3529 & $2.57 \mathrm{e}-15$ & $4.42 \mathrm{e}-14$ & $1.45 \mathrm{e}-15$ & \\
HS 1312+3508 & $2.12 \mathrm{e}-15$ & $1.00 \mathrm{e}-13$ & & \\
HS 1340+3307 & $6.20 \mathrm{e}-16$ & $9.11 \mathrm{e}-15$ & & \\
HS 1614+4709 & $5.92 \mathrm{e}-14$ & $1.17 \mathrm{e}-15$ & $5.81 \mathrm{e}-15$ & \\
HS 1641+5053 & $1.37 \mathrm{e}-15$ & $1.73 \mathrm{e}-14$ & & \\
\hline
\end{tabular}

Table 8 .

\begin{tabular}{lll}
\hline \hline Galaxy & & Intensity $^{a}$ \\
\hline HS $0915+5540$ & D & \\
HS $1304+3529$ & D & 5 \\
HS $1312+3847$ & C & \\
HS $1312+3508$ & D & 4 \\
HS $1424+3836$ & D & 9 \\
HS $1442+4250$ & C & \\
HS 1507+3743 & D & 6 \\
HS $1657+5735$ & D & 4 \\
HS $1728+5655$ & C & \\
\hline
\end{tabular}

${ }^{a}$ Dereddened line fluxes relative to $I(\mathrm{H} \beta)=100$.

we used the data from Table 9 to plot the metallicity - luminosity relation of star forming dwarf galaxies, as given by Skillman et al. (1989). Since the dwarf galaxies used by Skilmann et al. are all dwarf irregular galaxies, we further added some BCD's from Thuan et al. (1997). Our data distribute in the same region as the Thuan et al. 
Table 9. Candidates for very low metallicity galaxies

\begin{tabular}{lll}
\hline \hline & & \\
Galaxy & $12+\log (\mathrm{O} / \mathrm{H})$ & $M_{B}$ \\
\hline HS $1222+3741$ & 7.64 & -17.67 \\
HS $1236+3937$ & 7.47 & -15.67 \\
HS $1304+3529$ & 7.66 & -17.38 \\
HS $1318+3239$ & 7.81 & -17.22 \\
HS $1319+3224$ & 7.59 & -15.3 \\
HS $1330+3651$ & 7.66 & -17.15 \\
HS $1347+3811$ & 7.94 & -15.30 \\
HS $1424+3836$ & 7.97 & -16.06 \\
HS $1440+3120$ & 7.91 & -17.4 \\
HS $1442+4250$ & 7.89 & -14.73 \\
HS $1507+3743$ & 7.74 & -17.58 \\
\hline
\end{tabular}

Table 10. Frequency distribution of the ELG types in the University of Michigan survey (UM) and in our sample

\begin{tabular}{lrr}
\hline \hline Type & $\begin{array}{r}\text { this paper } \\
{[\%]}\end{array}$ & $\begin{array}{r}\text { UM } \\
{[\%]}\end{array}$ \\
\hline & & \\
SS & $4.8 \pm 2.4$ & 9.9 \\
DHIIH & $39.3 \pm 6.8$ & 29.7 \\
HIIH & $26.2 \pm 5.6$ & 24.8 \\
DANS & $14.3 \pm 4.1$ & 12.4 \\
SBN & $8.3 \pm 3.1$ & 13.2 \\
IP & $7.1 \pm 2.9$ & 9.9 \\
\hline
\end{tabular}

BCDs, with a similar amount of scatter and are - within the errors - in good agreement to the relation proposed by Skillman et al. (1989) (see Fig. 2). We conclude that the errors in our preliminary $12+\log (\mathrm{O} / \mathrm{H})$ determinations are small enough to indicate the existence in our sample of some interesting low-abundance BCDs. However, we cannot exclude a small offset in our $\mathrm{O} / \mathrm{H}$ scale.

\section{The properties of the sample}

In this section we present some statistics on the relevant spectroscopic parameters of the sample as well as of subsamples of different morphological subtypes.

Our sample was selected in certain fields which contain huge underdensities. One concern is that its galaxy content is thus different from the galaxy content of samples selected from the general field environment. In passing we should note that Popescu et al. (1997) found that most of our sample galaxies belong to the normal field features of the large scale structure. Nevertheless, we make an extra check, by comparing the frequency distribution of the Salzer et al. (1989b) types (see Tables 3, 5, and 6) with those from the University of Michigan (UM) survey, which is a general field survey. The comparison is given in Table 10 and indicates little significant differences in the type distribution of the two samples. If at all, we have some more DHIIH galaxies and fewer SBN objects in our sample. This is to be expected due to the selection criteria we adopted, namely bright objects were excluded from the survey (Popescu et al. 1996). The motivation was that bright objects were already included in other catalogues and we were mainly interested in dwarf galaxies. Since the frequency distribution of different morphological types is very close to that of the UM survey, we also expect a correlation between the Salzer et al. (1989) morphological types of our objects and their absolute blue magnitude. We verified that this correlation exists, but with a reasonable scatter in each sub-class. This scatter (peak-to peak) can be as large as 4 mag in most of the type bins. For a detailed discussion of the sample properties as a function of the galaxy density see Popescu et al. (1999).

The histogram of the $\mathrm{H} \beta$ luminosities of the whole sample is given in Fig. 3a. The luminosities were calculated for a Hubble constant $H_{0}=75 \mathrm{~km} \mathrm{~s}^{-1} \mathrm{Mpc}$ and they were corrected for internal extinction. The distribution is asymmetric, with a sharp cut-off towards the brighter end. This is due to the selection criteria we adopted, as mentioned above (see also Popescu et al. 1996). The incompleteness at the bright end is also obvious when compared with the $\mathrm{H} \beta$ distribution of other similar samples from the literature. For example, in Fig. 3d we show, for comparison, the $\mathrm{H} \beta$ luminosity distribution of a sample of 99 ELGs (Salzer et al. 1989b) from the UM objectiveprism survey and in Fig. 3e the same distribution from the ELG sample of Terlevich et al. (1991). In comparison with our distribution, the latter ones are more symmetrical and span over more orders of magnitude. They include at the bright end also Seyfert galaxies, which were not included in our study. The $\mathrm{H} \beta$ luminosity distributions of different morphological subtypes are shown in Fig. 3b (for SS and DHIIH galaxies) and in Fig. 3c (DANS and HIIH), respectively. The differences between them show the known trend of increasing $\mathrm{H} \beta$ luminosities from the SS to DANS class, though a significant overlap exist, too.

The expected correlation between the $\mathrm{H} \beta$ luminosities and the blue magnitudes is shown in Fig. 3f, where different morphological subtypes are plotted with different symbols. A linear least square fit to the $\log (L(\mathrm{H} \beta))=f\left(M_{B}\right)$ data (plotted with solid line) gives a slope of -0.388 and a correlation coefficient of 0.85 . This is close to the the slope $(-0.391)$ of a similar correlation found by Salzer et al. (1989b) for the ELGs of the Michigan Survey. The slope of -0.391 was derived when the Seyfert and SS galaxies were excluded from the correlation. This is consistent with our set of data, which does not contain Seyfert galaxies. Also the SS galaxies of our sample seem to follow in a better way the correlation, while those found by Salzer are lying mainly in the upper part of the correlation. As remarked by Salzer, this slope suggests that, within the 


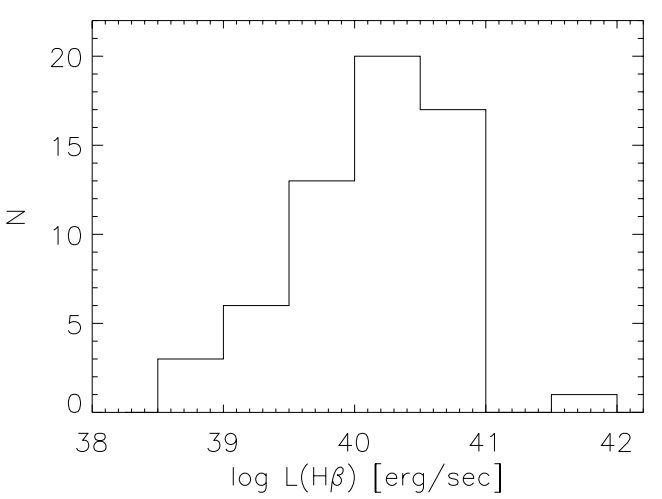

a)

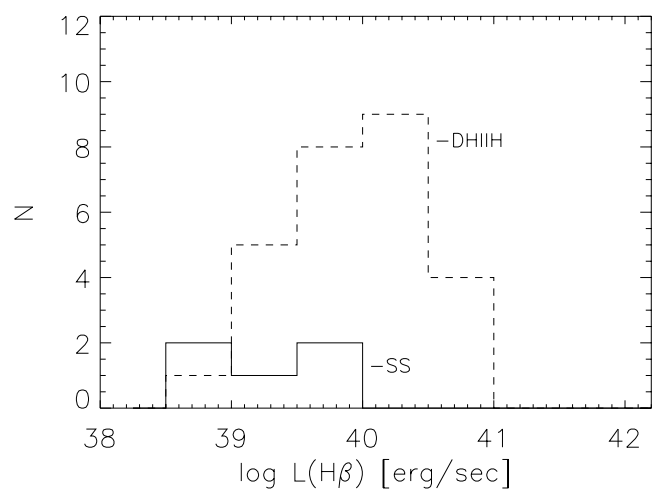

b)

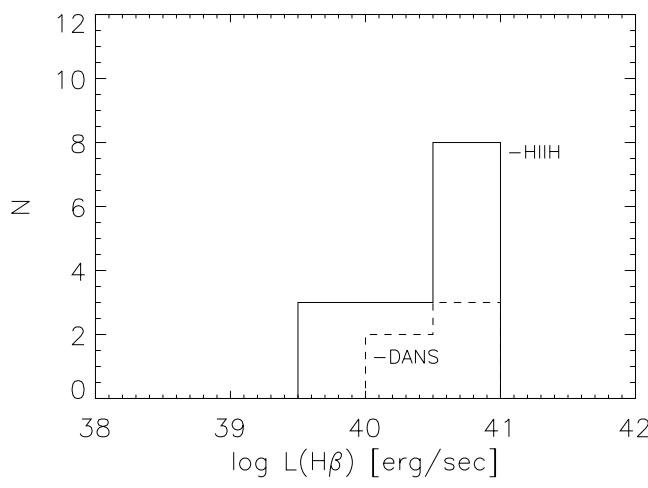

c)

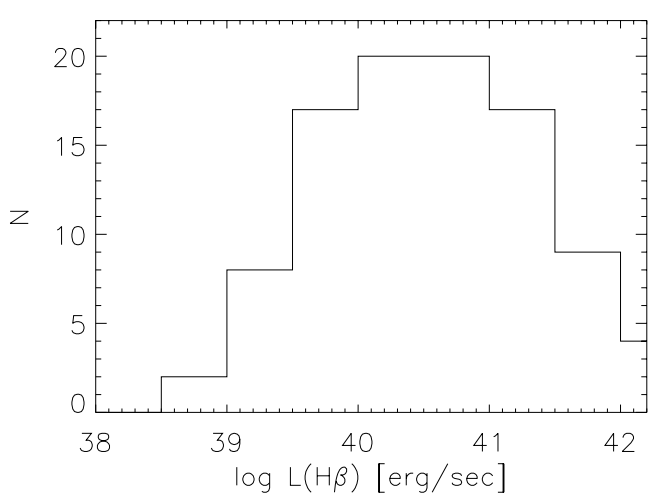

d)

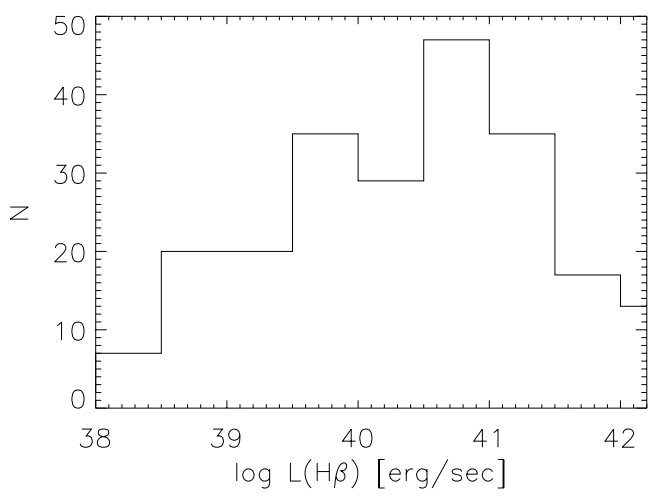

e)

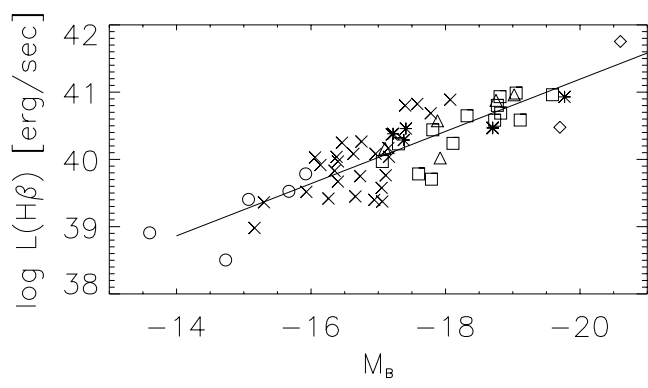

f)

Fig. 3. The distribution of the $\mathrm{H} \beta$ luminosities (corrected for internal absorption for: a) the whole sample; b) SS and DHIIH galaxies; c) HIIH and DANS galaxies; d) the sample of Salzer et al. (1989); e) the sample of Terlevich et al. (1991); f) The logarithm of the $\mathrm{H} \beta$ luminosities as a function of blue absolute magnitude. The different symbols indicate the ELG type: $\circ$ - Searle-Sargent Galaxy, $x$ - Dwarf HII Hotspot Galaxy, $\square$ - HII Hotspot Galaxy, $\triangle$ - Dwarf Amor. Nuclear Starburst, $\diamond$ Starburst Nucleus, * - Interacting Pair. The solid line represent a linear least-square fit to the data

uncertainties, the $\mathrm{H} \beta$ luminosity scales directly with the blue luminosity: $L(\mathrm{H} \beta) \propto L_{B}$. This would indicate that the recent star-formation (in the last $\sim 10 \mathrm{Myr}$ ) and the integrated star formation are related. On the other hand the scatter from the correlation suggests variations in the global equivalent widths, $W(\mathrm{H} \beta)$, which means different stages of activity. If the total blue magnitude is a good measure of the average past star formation, then the scat- ter indicates an intrinsic variation in the ratio between the present star formation rate and the average past.

The distribution of the $\mathrm{H} \beta$ equivalent widths is also given in Fig. 4.

\section{Appendix}

During our survey for emission line galaxies we also identified a few quasars and AGNs. Here we give some 


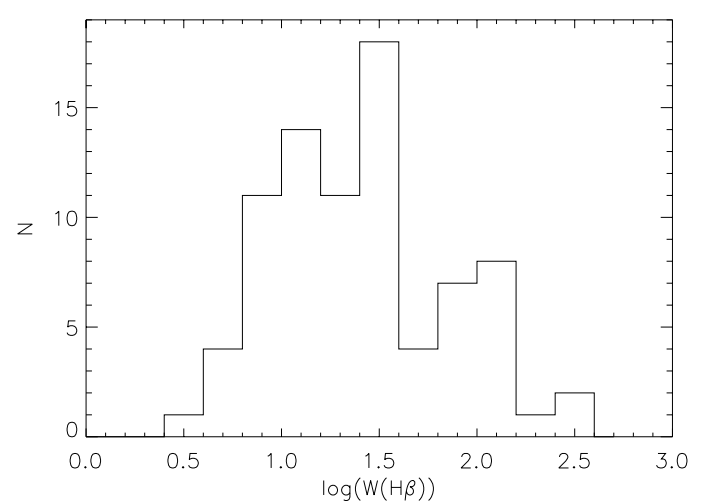

Fig. 4. The distribution of the $\mathrm{H} \beta$ equivalent widths (in units of $\AA$ )

spectroscopic information about a peculiar QSO found in our survey, namely HS $1643+5313$. This object looked peculiar also in the objective-prism scans, in the sense that it displayed a double peaked feature, just at the green head of the objective prism spectrum. A first possibility would

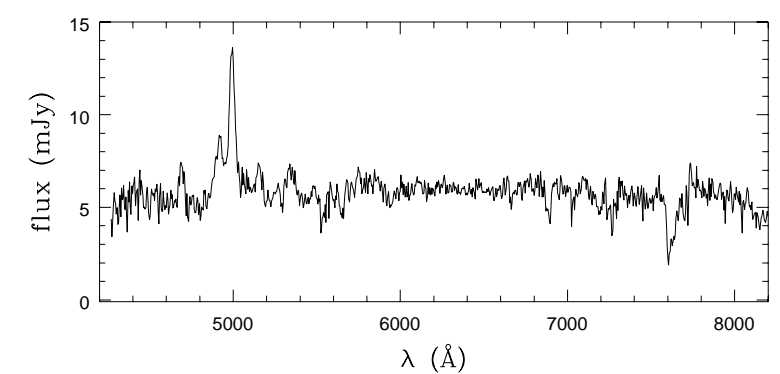

Fig. 5. Slit spectrum of the quasar HS $1643+5313, z=0.785$

have been that the strongest feature were the $[\mathrm{OIII}] \lambda 5007$ line of a narrow-emission line galaxy while the second feature a cosmic or only noise. No other combination of emission-lines at any redshift and at this dispersion were known to fit the above mentioned spectrum. The spectrum was therefore chosen mainly with the hope of being still a narrow-emission line galaxy. The slit spectrum (Fig. 5) displays the same combination of a double emission-line feature. After analysing in detail the spectrum as well as its direct image we reached the conclusion that the object is a QSO at $z=0.785$, the emission-features being thus only one line, namely the MgII $\lambda \lambda 2798$ blend, but with an absorption dip inside. There is also some faint detection of FeII at $5262 \AA$, but the $\mathrm{S} / \mathrm{N}$ ratio of the spectrum is too poor for a clear detection of further fainter lines. A high resolution spectrum of the near infrared region would clarify whether the absorption is internal to the QSO or arises from a foreground cloud or galaxy.

Acknowledgements. U.H. acknowledges the support of the SFB 375. This research has made use of the NASA/IPAC Extragalactic Database (NED) which is operated by the Jet Propulsion Laboratory, California Institute of Technology, under contract with the National Aeronautics and Space Administration.

\section{References}

Allen D.A., Wright A.E., Goss W.M., 1976, MNRAS 177, 91 Aloisi A., Tosi M., Greggio L., 1999, AJ 118, 302

Binggeli B., Sandage A., Tammann G.A., 1985, AJ 90, 1681

Brocklehurst M., 1971, MNRAS 153, 471

Burstein D., Heiles C., 1984, ApJS 54, 33

de Lapparent V., Geller M.J., Huchra J.P., 1986, ApJ 302, L1

Dopita M.A., Evans I.N., 1986, ApJ 307, 431

Gallagher J.S., Hunter D.A., 1989, AJ 98, 806

Garnett D.R., Skillman E.D., Dufour R.J., Shields G.A., 1997, ApJ 481, 174

Hagen H.-J., Groote D., Engels D., Reimers D., 1995, A\&AS 111,195

Howarth I.D., 1983, MNRS 1983, 203, 301

Izotov Y.I., Thuan T., 1999, ApJ 511, 639

Kunth D., Contini T., 1998, in "Wolf-Rayet Phenomena in Massive Stars and Starburst Galaxies", van der Hucht K.A., Koenigsberger G., Eenens P.R.J. (eds.), IAU Symp. 193. San Francisco: ASP (in press)

Kunth D., Maurogordato S., Vigroux L., 1988, A\&A 204, 10

Lequeux J., Peimbert M., Rayo J.F., Serrano A., TorresPeimbert S., 1979, A\&A 80, 155

Lynds R., Tolstoy E., O’Neil E.J., Hunter D.A., 1998, ApJ 116, 146

Loose H.-H., Thuan T.X., 1986, ApJ 309, 59

Masegosa J., Moles M., Del Olmo A., 1991, A\&A 244, 273

Mathis J.S., Rosa M.R., 1991, A\&A 245, 625

McCall M.L., Rybski P.M., Shields G.A., 1985, ApJS 57, 1

Oke J.B., 1990, AJ 99, 1621

Osterbrock D.E., Cohen R.D., 1982, ApJ 261, 64

Popescu C.C., Hopp U., Elsässer H., 1997, A\&A 328, 756

Popescu C.C., Hopp U., Rosa M., 1999, A\&A 350, 414

Popescu C.C., Hopp U., Hagen H.J., Elsässer H., 1996, A\&AS 116,43

Popescu C.C., Hopp U., Hagen H.J., Elsässer H., 1998, A\&AS 133, 13

Pustilnik S.A., Thuan T.X., Brinks E., Lipovetsky V.A., Izotov Y.I., 1999 (in preparation)

Salzer J.J., MacAlpine G.M., Boroson T.A., 1989a, ApJS 70, 447

Salzer J.J., MacAlpine G.M., Boroson T.A., 1989b, ApJS 70, 479

Sargent W.L.W., Searle L., 1970, ApJ 162, L155

Schaerer D., Contini T., Pindao M., 1999, A\&AS 136, 35

Schulte-Ladbeck R.E., Crone M.M., Hopp U., 1998, ApJ 493, L23

Schulte-Ladbeck R.E., Hopp U., Crone M.M., Greggio L., 1999, ApJ 525, 709

Skillman E.D., Kennicutt R.C., Hodge P.W., 1989, ApJ 347, 875

Telles E., Melnik J., Terlevich R., 1997, MNRAS 288, 78

Terlevich R., Melnick J., Masegosa J., Moles M., Copetti M.V.E., 1991, A\&AS 91, 285

Thuan T.X., Izotov Y.I., Lipovetsky V.A., 1997, ApJ 477, 661

Vennik J., Hopp U., Popescu C.C., 2000, A\&AS (submitted)

Vílchez J.M., 1995, AJ 110, 1090

Whitford A.E., 1958, AJ 63, 201 